\title{
EV ALUACIÓN DE LA IMPLEMENTACIÓN DE LA POLÍTICA PÚBLICA DE PRIMERA INFANCIA EN LA MODALIDAD DE ENTORNO INSTITUCIONAL EN EL MUNICIPIO DE NENA
}

\author{
LAURA CÓRDOBA MUÑOZ \\ Licenciada en Pedagogía Infantil \\ Magister en Educación \\ Universidad Surcolombiana
}

\section{¡Sobre el Problema ...!}

La Primera Infancia, comprendida entre los cero y los seis años, es una etapa en la que tanto los niños como las niñas requieren estimulación, atención y tratamiento especiales que les permita desarrollarse biológica, psicológica, cultural y socialmente. Diversos estudios realizados desde distintas disciplinas han comprobado con argumentos sólidos que desde su concepción los niños cuentan con una serie de capacidades físicas, psicológicas, emocionales y sociales que facilitan el aprendizaje, la socialización y la comunicación.

De acuerdo con lo anterior, existen muchos niños que tienen la posibilidad de crecer en un ambiente familiar donde son protegidos, cuidados, atendidos, reciben afecto y carino, tienen acceso a servicios de salud, alimentación balanceada, son estimulados y asisten a jardinos infantiles. Sin embargo, a diferencia de esos niños, existen otros que no tienen las mismas posibilidades, debido a la situación económica o estructural de sus familias y al contexto sociocultural al que pertenecen.

En ese sentido, de manera progresiva se ha ido consolidando tanto a nivel internacional como nacional un marco normativo que regula lo concerniente a la primera infancia y que intenta garantizar sus derechos y comprometer al Estado en lo que respecta al diseño, la ejecución, la implementación, la evaluación y el seguimiento de las políticas que involucren a la Primera Infancia.

En Colombia, en el año 2007 el Consejo Nacional de Política Económica y Social - CONPES, aprobó la Política Pública Nacional de Primera Infancia - "Colombia por la Primera Infancia" la cual define como objetivo general "Promover el desarrollo integral de los niños y niñas desde la gestación hasta los 6 años de edad; respondiendo a sus necesidades y características especificas, y contribuyendo ași al logro de la equidad e inclusión social en Colombia". Del mismo modo, hace referencia a tres modalidades de atención familiar, comunitaria e institucional, yen una de sus diez líneas estratégicas hace alusión a realizar seguimiento y evaluación a la implementación y el impacto de la politica en los níños y niñas de 0 a 6 años.

Para la implementación de la política se creó el documento "Guía Operativa para la prestación del servicio de atención integral a la Primera Infanciar" en donde se describe cada una de las modalidades de atención, se plantea el esquema de funcionamiento y los requerimientos básicos para la atención de acuerdo con cada modalidad. 
Con el fin de tener en cuenta las problemáticas de la Primera Infancia y de atender a los niños y niñas menores de seis años que no cuentan con toda la atención que se requiere en esta edad, dicha política pública de primera inf ancia se implementa en el municipio de Neiva desde el año 2008 y de manera periódica las personas delegadas por la Alcaldía, la Gobernación y el Programa de Primera Inf ancia han presentado informes parciales que dan cuenta de cifras relacionadas con la cobertura, la edad de los nin̄os, el número de menores que han logrado beneficiarse del programa, así como también consignan información relacionada con la edad de los docentes, el nivel de formación, el número de hijos y su estado civil. De acuerdo con lo anterior, es evidente que la información suministrada en dichos informes no es detallada y precisa en lo que concierne al proceso de implementación de la política pública nacional de Primera Infancia en el municipio de Neivar así como también es notorio que el enfoque que se le otorga a los seguimientos es significativamente superficial $y$. por lo tanto, no profundiza en detalles del proceso y se centra principalmente en cifras muy generales.

Si bien referirse a la implementación hace parte del desarrollo de un proceso, los informes así no lo mencionan y, por el contrario, es bastante notoria la carencia de un seguimiento detallado y puntual que nose limite exclusivamente a dar a conocer lo que concierne a la cobertura o referirse de manera ligera a otros aspectos.

En ese sentido, se desconocen las problemáticas que se están atendiendo, las actitudes que caracterizan al personal que atiende dicha población, la preparación y formación que reciben para atender a los menores, el tipo de acompañamiento que se brinda a los niños y niñas, la planeación y las estrategias que se utilizan para el desarrollo de las actividades pedagógicas, los materiales y recursos que se emplean, los escenarios con que se cuenta para atender a los menores, el tipo de relación que se da con las familias, las fortalezas y debilidades de la implementación.

De acuerdo con lo expuesto, se planteó el siguiente interrogante que orientó el proceso de investigación:

¿Cómo se ha desarrollado el proceso de implementación de a Modalidad de entorno institucional de la Política Pública Nacional de Primera Infancia en el municipio de Neiva? con el interés de desarrollar un proyecto de investigación que muestre a la sociedad la importancia de comprender que la Primera Infancia es una etapa fundamental en el desarrollo de cualquier persona, y por ende que las acciones que se llevan a cabo con esta población deben considerarse trascendentales y requieren ser adelantadas de una manera seria y rigurosa que les permita a los niños y niñas participar en procesos que aporten a su desarrollo integral.

En ese sentido, la ejecución del proyecto permitió destacar que no basta con que se desarrollen diferentes actividades o procesos, si no existe algún tipo de seguimiento que permita contribuir con el proceso que se ha venido adelantando en diferentes escenarios y contextos encaminados a otorgar a la primera inf ancia la importancia que requiere. 
Así mismo, evaluar el proceso de implementación de la modalidad de entorno institucional de la política pública de Primera Infancia en el municipio de Neiva, facilitó dar a conocer cómo se ha adelantado este proceso, qué actividades se están desarrollando, identificar fortalezas y debilidades, destacar los logros alcanzados, definir los aspectos que deben mejorarse, replantearse o consolidarse, cuales son las problemáticas que se estấn atendiendo especificamente en el municipio de Neiva, debido a que los informes presentados anteriormente no relacionan lo expuesto y centran su atención en el número de niños atendidos y en la cantidad de docentes que pertenecen ai Programa sin otorgar mayor atención a las actividades y demás aspectos del proceso.

Consecuencia de lo anterior, iniciar con un proceso de evaluación a la implementación de la política pública nacional de Primera Infancia, específicamente en la modaiidad de entorno institucional, desde una mirada distinta a la que se ha venido presentando se convirtió en una alternativa válida y posible para debilitar la distancia existente entre lo que se dice y lo que se hace aś como aportar a la detección de falencias y fortalezas en cuanto al proceso de implementación y de los ajustes que deben hacerse para que ei trabajo que se viene adelantando sea reconocido por su caildad, por otorgar mayores y mejores oportunidades a a Primera Infancia y por los diferentes aportes que haga.

Teniendo claridad sobre la problemática a tratar y sobre la necesidad e importancia de investigarla el objetivo que orientó el desarroilo de la investiggación fue evaiuar el proceso de implementación de b modalidad de entorno institucional de la política pública nacional de Primera Inf ancia en el municipío de Neiva quegarantice que su implementación y desarroilo responda a las exigencias de pertinencia social de la misma.

Del mismo modo, se hizo una elaboración permanente dei marco teórico que tuvo básicamente como referentes las políticas públicas y el proceso de implementación de éstas; y de otra parte los diferentes procesos, experiencias y actividades que involucran a la Primera Inf ancia en el contexto internacional, nacional y locai desde diversas perspectivas y que destacan la importancia de atender y estudiar esta etapa.

\section{¿Cómo se levó a cabo el proyecto de investigación?}

Teniendo en cuenta que la evaluación del proceso de implementación de la modalidad de entorno institucional de la Política de Primera Infancia en el municipio de Neiva implica tener en cuenta una variedad de factores que inciden de manera permanente en este proceso y lo hacen comple jo y cambiante, el enfoque de esta investiggación es de tipo cualitativo, la cual se asume

... "como un proceso que se alimenta continuamente, de yen, la confrontación permanente de las realidades intersubjetivas que emergen a través de la interacción dei investigador con los actores de los procesos y reaiidades socio-cuiturales y personales objeto de análisis así como del análisis de la documentación teórica, pertinente y disponib|e" (Sandoval, 199ó). 
De acuerdo con lo anterior, la investigación se inició con un proceso de acercamiento a los sujetos que hacen parte de la realidad y de la problemática que fue objeto de análisis yestudio; con el fin de interactuar con cada uno de ellos y establecer comunicación, contacto e interacción permanentes, lo cual faciütó conocer cadáa uno de ios elementos que constituyen su contexto y su realidad.

Así mismo, el análisis de documentos se asumió como una estrategia fundamental que influyó de manera determinante en la definición de la problemática a investigar y que de igual forma se convir tió en una herramienta para abordar y comprender ia reaiidad.

Abordar la realidad desde la perspectiva expuesta no significó"... la renuncia a lo numérico o cuantitativo, sino más bien, la reivindicación de lo subjetivo, lo intersubjetivo, lo significativo y lo particular, como prioridades de análisis para ia comprensión de ia realidad social". (Sandoval 1996).

Por lo mencionado, en algunas etapas o momentos del desarrollo del trabajo de investigación el uso de algunas cifras o datos numéricos sirvió como elemento para explicar y comprender determinadas situaciones sin que esto se convirtiera en el eiemento central del análisis y la comprensión de los sujetos y la reaiidad de la que hacen parte.

En concordancia con lo anterior, et método de investigación que sustentó el desarroilo del proyecto fue b etnografía, ia cual "consiste en descripciones detalladas de situaciones, eventos, personas, interacciones y comportamientos que son observables. Incorpora lo que los participantes dicen, sus experiencias, actitudes, creencias, pensamientos y reflexiones tal como son expresadas por ellos mismos y no como uno los describe" (González y Hernández, 2003).

De acuerdo con lo anterior, b etnografía permitió evaiuar el proceso de impiementación de la modalidad de entorno institucionai de ia Poítica Pública Nacional de Primera infancia en et municipio de Neiva, teniendo como eiemento principai ias percepciones, vivencias, experiencias y comportamientos de ios padres de famiiia, niños, niñas, auxiliares de cuidado, agentes educativos, personal administrativo y de apoyo que hacen parte de ias sedes Los Almendros, Chapinero yEl Jardin.

Fue necesario definir una serie de instrumentos de investigación (análisis de documentos, entrevistas a profundidad y registros de observación) que hicieron posible la recoiección de la información y et acercamiento constante a< docentes, auxiliares, miembros del equipo interdiscipiinario de apoyo y padres de familia para comprender y analizar ia problemática abordada.

E proceso que se reaiizó con las entrevistas a profundidad fue diferente al trabajo de análisis de documentos y registros de observación el cual constó de tres momentos: eiaboración, validación y aplicación. Una vez fueron elaboradas ias entrevistas se procedió a validarias con per sonai que laboraba para ia modaiidad de entorno institucional en Neiva que no hizo parte de ia muestra con el fin de conocer b validez y confiabilidad del instrumento en el 
momento de su aplicación. Este proceso de validación contó con sugerencias, recomendaciones y aportes de las personas entrevistadas, que permitieron precisar los aspectos necesarios y aprobar la versión definitiva de las entrevistas.

Realizada la validación, se aplicaron de manera personal las entrevistas a la muestra seleccionada con el fin de garantizar la calidad del proceso de recolección de la información.

Para el diseño de los registros de observación el referente que se utilizó fue la Guía Operativa para la Prestación del Servicio de Atención Integral a la Primera Infancia. Elaborados los registros de observación se aplicaron de manera directa en cada una de las sedes que se escogieron para realizar la presente investigación.

La población que fue objeto de estudio y análisis es la que pertenece a los centros de atención (Sede Los Almendros, Sede Chapinero, Sede E Jardín y Sede Urdaneta) de la modalidad de entorno institucional en el Municipio de Neiva de la Política Pública Nacional de Primera Inf ancia.

La muestra que hizo parte del proyecto de investigación corresponde al equipo interdisciplinario de apoyo, a los agentes educativos, a las auxiliares de cuidado y a los padres de familia de tres de los centros de atención de la modalidad de entorno institucional los cuales son: Sede Chapinero, Sede Los Almendros y Sede E Jardín.

Del equipo interdisciplinario de apoyo de la modalidad de entorno institucional: se entrevistó al psicólogo y al nutricionista; igualmente se entrevistó a la Coordinadora Pedagógica responsable de la coordinación de la modalidad de entorno institucional, los agentes educativos (ó docentes de la Sede de Chapinero, 4 de la Sede El Jardín y 4 de la Sede los Almendros para un total de 14 docentes); las Auxiliares de cuidado (3) y los padres de familia (12 padres de las distintas sedes de la modalidad de entorno institucional).

De acuerdo con los objetivos propuestos y para la recolección y el análisis de la información se definieron cuatro categorías de análisis (Preparación para la prestación del servicio, Cuidado y educación inicial, Salud y Trabajo con Padres de Familia) que permitieron evaluar el proceso de implementación de la modalidad de entorno institucional de la Política de Primera Infancia en el municipio de Neiva:

\section{¿Qué se encontró?}

E contacto permanente con el personal que integra las sedes de atención Los Almendros, Chapinero y $\mathrm{E}$ Jardín de la modalidad de entorno institucional de la Política Pública Nacional de Primera infancia en el municipio de Neiva permitió conocer las percepciones, opiniones, experiencias y puntos de vista de docentes, auxiliares, padres de familla, coordinadora pedagógica, nutricionista y psicólogo y conocer los procesos que se vienen desarrollando en cada una de las sedes. Este acercamiento fue vital para el desarrollo de la investigación y especificamente para la evaluación de la implementación de la Política Pública Nacional de Primera Infancia en Neiva. 
A continuación se presenta los principales hałlazgos del proceso de investigación desarrollado:

- En el municipio de Neiva se viene implementando la modalidad de entorno institucional de Primera Inf ancia desde el año 2008, atendiendo población menor de 5 años de edad que induye niños y niñas de estratos 1 y 2 de algunos barrios y asentamientos de la Comuna 4 del municipio de Neiva.

La modalidad de entorno institucional nose ofrece en ninguna de las otras comunas del municipio, a pesar de que existen ó comunas, integradas por algunos barrios en donde hay población infantil menor de 5 años de edad que requiere atención en nutrición, cuidado y educación inicial.

Razón por la cual se puede afirmar que la implementación de la modalidad de entorno institucional de la Política de Primera Infancia en Neiva es restringida en cuanto a los sectores que se están beneficiando del Programa.

- El proceso de preparación que se adelanta para la implementación de la modalidad de entorno institucional de la Política de Primera Infancia en Neiva consta de las siguientes etapas: trámites legales, que incluyen firma de contratos, gestión de recursos y diligenciamiento de documentos; adecuación de los centros infantiles (sedes) o planta física para el ofrecimiento de la modalidad que contempla dotación de materiales y recursos para el funcionamiento del programa; conformación del equipo interdisciplinario de apoyo (nutricionista, coordinadora pedagógica y psicólogo) y selección del personał docente y auxiliar encargado de atender a los menores y desarrollar las actividades de cada componente; capacitación al personal encargado de atender a los menores acerca de Primera Infancia; promoción y divulgación del programa; ubicación de los niños y niñas y realización del proceso de matrícula.

- Las actividades que se llevan a cabo con la población beneficiada hacen parte de tres componentes de atención los cuales son: nutrición, cuidado y educación inicial. En materia de nutrición, los centros infantiles de atención a la modalidad de entorno institucional suministran a los niños y niñas un almuerzo y dos refrigerios diarios (uno en la mañana y otro en la tarde) que aportan a la alimentación diaria de los menores, a su estado nutricional y a la formación de hábitos alimenticios, puesto que para el programa el estado nutricional de los niños y niñas beneficiados es decisivo en los procesos de aprendizaje yen el desempeño de los menores.

En cuanto al cuidado y la educación inicial la interacción entre docente y auxiliares con los niños y niñas está mediada por et juego a través de la estrategia pedagógica denominada Momentos que busca principalmente que los menores se socialicen con sus compañeros y desarrollen habilídades artisticas, cognitivas, motoras y de lenguaje que los preparen para el nivel preescolar.

E programa desarrolla actividades a partir de cuatro Momentos los cuales son: Vamos a crear, Vamos a explorar, Vamos a construir y Vamos a jugar. 
- El personal encargado de atender a la población que se beneficia de la modalidad de entorno institucional en las sedes (Los Almendros, Chapinero y El Jardín) de Primera Infancia en Neiva estáa integrado por un equipo interdisciplinario de apoyo al que pertenecen el psicólogo, el nutricionista y la coordinadora pedagógica, así como también las docentes y auxiliares docentes.

Igualmente, se encontró que el apoyo de las auxiliares docentes no es permanente en las sedes Los Almendros, Chapinero y $\mathrm{E}$ Jardín debido a que el tiempo de vinculación de las auxiliares da cuenta de que se han vinculado recientemente al Programa. Así mismo, se pudo evidenciar que no existe un auxiliar de cuidado por cada 40 niños tal como lo precisa la Guía Operativa de atención. Lo anterior deja en evidencia la carencia de auxiliares en los Centros Infantiles de la modalidad de entorno institucional.

En relación con el equipo interdisciplinario de apoyo, la modalidad de entorno institucional del programa cuenta con la disponibilidad de psicólogo, nutricionista y coordinador pedagógico; sin embargo, los tres profesionales que integran el equipo no son suficientes de acuerdo con el número de niños y niñas que se vienen atendiendo en las sedes.

Respecto a la formación profesional del personal que hace parte de la modalidad de entorno institucional se encontró que predominan las personas que cuentan con título de licenciadas en diferentes áreas del conocimiento que poseen experiencia en el trabajo con población de los diferentes niveles del sistema educativo, siendo muy reducido el personal que cuenta con titulo de especialista.

- En lo que compete a las sedes Los Almendros y Chapinero de la modalidad de entorno institucional en Neiva, estas funcionan en casas que se han habilitado para la atención de los niños y niñas, que cumplen con las condiciones mínimas de calidad, pero no están dotadas de espacios amplios para el juego libre. Por su parte la Sede El Jardín funciona en el Colegio Gimnasio Moderno que es el operador del servicio en el municipio de Neiva y, de acuerdo con lo que se observó, es la única que cuenta con espacios amplios o zonas deportivas.

En relación con la dotación de materiales de las sedes Los Almendros, Chapinero y El Jardín, éstas cuentan con los recursos suficientes para el desarrollo de las actividades relacionadas con el cuidado y la educación inicial, así como también para la preparación y suministro de alimentos, cumpliendo con lo requerido por el Programa para ofrecer la modalidad de entorno institucional.

- En relación con el periodo anual que se destina para la atención a los niños y niñas seencontró que no existe un periodo definido. De acuerdo con la información suministrada por los padres defamilia, las docentes, las auxiliares y el equipo interdísciplinario de apoyo, la atención que se brinda no contempla un período determinado; por el contrario, el término de duración de la prestación del servicio varía cada año debido a que la modalidad de atención de entorno institucional de la Política de Primera Infancia inicia una vez se han adelantado las gestiones administr ativas de orden nacional que le permiten al operador del servicio iniciar con sus labores. La ausencia de un período de atenciór 
defínido por año deja a los menores beneficiados por el Programa durante varios meses del año sin ningún tipo de atención en cuanto a nutrición, educación inicial y cuidado, lo que ocasiona que su proceso de formación se vea interrumpido constantemente.

\section{¿Qué se propone?}

La evaluación de la implementación de la modalidad de entorno institucional de la política de primera infancia en el múnicipio de Neiva evidencia que no existe un período determinado de atención, el número de profesionales que atiende a los menores no es el adecuado, la concepción de formación y evaluación es restringida, los centros de atención cuentan con espacios reducidos para el juego libre y no existen sedes en lugares altamente vulnerables del múnicipio.

Lo anterior muestra que algunos aspectos del proceso de implementación nose están adelantando de manera adecuada y no dan cumplimiento a los requerimientos consignados en la Gúa Operativa para la prestación del servicio. Así mismo, la situación encontrada refleja que existe distancia entre lo que se plantea en el documento guía para la implementación de la modalidad de entorno institucional y algunas acciones que se adelantan en las sedes de primera infancia.

En ese sentido, es necesario definir un período anual de atención para atender a los menores que se vinculen a la modalidad de entorno institucional, con el fin de garantizarles los espacios pedagógicos necesarios para el desarrollo de los componentes de atención que ofrece el programa. lgúalmente, esto permite que los menores hagan parte de espacios de socialización e interacción qúe contribuyen a su desarrollo integral debido a que su condición económica u otras dificultades son limitantes que impiden el acceso a otros espacios de formación dirigidos a la Primera Infancia.

Además de establecer un período de atención, es indispensable que el operador del servicio integre al equipo interdisciplinario de apoyo el número de coordinadores, nutricionistas y psicólogos correspondiente al número de niños y niñas atendidos, con el fin de ofrecer atención oportuna y de calidad que permita detectar las necesidades de los menores y ofrecer atención y orientación a las familias.

Por otra parte, es necesario queen cada una de las sedes de primera infancia de la modalidad de entorno institucional se incluya un auxiliar de cuidado por cada grupo de niños que, en alianza con a docente titular del curso, apoye batención, interact tíe con ellos y promueva espacios de socialización y aprendizaje que contribuyan con su proceso de formación. Esta inclusión de auxiliares en los grupos de atención debe realizarse desde el inicio del programa y mantenerse durante todo el proceso con el fin de garantizar apoyo y cuidado permanente a los menores de edad. Así mismo, el operador del servicio debe ser responsable de formar al personal que labora en los centros infantiles de la modalidad de entorno institucional. 
No es suficient a conformar un equipo de trabajo y realizar una jornada de capacitación; es importante que las personas que estấn a cargo de los niños y las niñas tengan espacios periódicos de formación que les permitan no sólo formarse en relación con la política de Primera Infancia sino también en relación con las problemáticas de esta etapay sobre la manera como éstas pueden ser abordadas desde las aulas o los centros infantiles. Los espacios de formación que se habiliten deben contar con la participación de personal competente y reconocido que domine lo concerniente al tema y que tenga la capacidad de orientar al personal y contribuir con su proceso de formación profesional.

Para fortalecer estos espacios de formación y contribuir con el mejoramiento continuo de las docentes, es importante que se transforme la concepción que se tiene de evaluación debido a que esta se considera como una estrategia para medir el desempeño de las docentes y auxiliares. Loque se requiere es que la evaluación sea vista como un espacio de reflexión y retroalimentación en donde cada docente pueda autoevaluarse y también pueda participar en la evaluación del desempeño de sus compañeros con el propósito de detectar los aspectos que deben mejorarse y las acciones que pueden realizarse para que esto se dé.

En relación con la planta física donde se vienen atendiendo a los menores que se han vinculado a la modalidad de entorno institucional, es necesario que se construyan centros infantiles o se preste el servicio en lugares que cuenten con espacios amplios para el juego, el esparcimiento, el deporte, la recreación, la realización de actividades comunes y el suministro de alimentos que estén notoriamente diferenciados y que puedan utilizarse exclusivamente para la finalidad que sean construidos.

Las sedes de la modalidad de entorno institucional deben extenderse a diferentes comunas del municipio de Neiva, en especial a aquellas en donde se encuentran asentamientos, barrios o sectores habitados por población infantil que requiere beneficiarse del programa y que por sus condiciones no tienen la posibilidad de acceder a una institución educativa o no estẩn siendo atendidos en otros programas dirigidos a la Primera Infancia.

Además de intervenir en los aspectos específicos que se mencionaron anteriormente, es necesario que el personal que hace parte de los centros infantiles evalúe periódicamente la forma como se están desarrollando las actividades que hacen parte de la implementación de la modalidad de entorno institucional en el municipio de Neiva.

Dicha evaluación requiere centrar su interés en el desarrollo de los procesos yen el impacto que éstos generen en los niños, padres de familia, docentes, auxiliares y miembros del equipo interdisciplinario de apoyo; pues no basta con ofrecerel programa y vincular los menores a la modalidad de entorno institucionali, sino adelantar las acciones necesarias para que los niños reciban una atención de calidad. Esta forma de asumir la evaluación rompe con el habitual interés y la preocupación constante por entregar informes que relacionen índices de cobertura, y se enfatiza en dar prioridad a aquellos aspectos que contribuyen con la formación de los niños y nin̄as de nuestro país. Lo anterior obedece a que la naturaleza de los procesos que se desarrollan con los menores y sus padres de familia no poseen unas dinămicas permanentes sino que, por el contrario, cambian de manera constante debiuo 
a las características particulares de cada uno de los sujetos que hacen parte de los centros infantiles (niños, niñas, padres de familia, docentes, auxiliares, coordinadora pedagógica, psicólogo y nutricionista), a su entorno y a las exigencias de orden nacional.

Para llevar a cabo la evaluación de las actividades que se realizan en la modalidad de entorno institucional en el municipio de Neiva, se propone conformar un Comité de Seguimiento, Asesoramiento y Evaluación integrado por un representante del equipo interdisciplinar io de apoyo, un delegado del operador del servicio (Colegio Gimnasio Moderno), un docente, un auxiliary un padre de familia de cada sede o centro infantil, que se reúna periódicamente.

En el Gráfico que se muestra a continuación se ilustra la forma como estará integrado el Comité de Seguimiento, Asesoramiento y Evaluación:

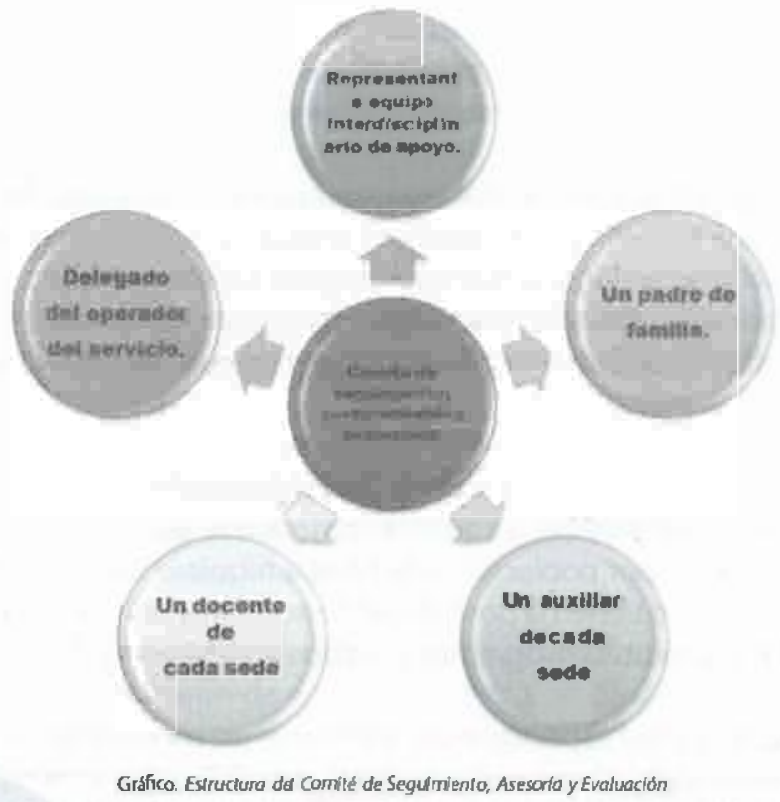

El Comité será responsable de evaluar las actividades, el proceso que se adelantó para el desarrollo de las actividades y los resultados de las mismas, con el fin de darlos a conocer a todo el personal que labora en los centros infantiles o sedes de la modalidad de entorno institucional de Primera Inf ancia.

De acuerdo a lo anterior, las funciones del Comité son las siguientes:

- Realizar seguimiento a cada una de las actividades que se llevan a cabo en los centros o sedes de la modalidad de entorno institucional de la Política Pública de Primera Inf ancia en el municipio de Neiva.

- Asesorar la planeación y preparación de las actividades que se adelanten en las sedes - centros infantiles de la modalidad de entorno institucional de la Política Pública de Primera Inf ancia en el municipio de Nieva. 
- Evaluar el desarrollo y los resultados de las actividades que se adelantan en cada uno de los centros infantiles o sedes de la modalidad de entorno institucional de la Política de Primera Inf ancia.

- Divulgar los resultados de la evaluación que realice a las actividades que se desarrollan en las sedes de la modalidad de entorno institucional en el municipio de Neiva.

De igual forma, el Comité de Seguimiento, Asesoramiento y Evaluación debe establecer que actividades requieren de estrategias alternativas para su mejoramiento y definir las acciones que se adelantaran para que esto se dé.

Además de definir las estrategias que se utilizarán para el mejoramiento de determinada actividad el Comité Evaluador debe hacer seguimiento a las estrategias y a la actividad con el fin de conocer los logros alcanzados, los aspectos en los que se mejoró y los que requieran algún tipo de seguimiento o acompañamiento permanente.

Para el caso de las actividades que requieren seguimiento, el Comité de Evaluación delegará a uno de sus miembros para que adelante este proceso, dependiendo de a actividad. Dentro de las actividades que el Comité tendrá en cuenta para et proceso de seguimiento, asesoramiento y evaluación se pueden relacionar las siguientes:

Divulgación del programa: en este aspecto, se tendrä en cuenta el proceso y las estrategias gue se utilicen para dar a conocer el Programa en los diferentes sectores, barrios y asentamientos del municipio de Neiva, y concientizar a los padres de familia sobre los beneficios de vincular a sus hijos o hijas al Programa.

Proceso de matrícula: en relación a este proceso, será de fundamental interểs para el Comité la recolección oportuna y completa de la documentación, el diligenciamiento de los formatos con información específica de los menores, la organización de b información y el acceso a esta.

Encuentros con padres de familia: para el caso de los encuentros con padres de familia se analizará la preparación, el desarrollo, los materiales, el lugar donde se lleven a cabo, la pertinencia de las temáticas abordadas, la asistencia, participación y observaciones de los padres de familia, el desempeño del personal encargado para el desarrollo de los mismos, el dominio de los temas tratados, y la frecuencia con que se realicen.

Formación de los docentes y auxiliar es: el Comité de seguimiento, asesoría y evaluación tendrá en cuenta los espacios de reflexión, análisis, discusión e interacción que se propicien en cada una de las sedes para la formación de los docentes y auxiliares, asi como también los materiales que se utilicen, los temas que se aborden, la participación de los docentes, la periodicidad de los encuentros y las estrategias que se implementen para el desarrollo de las actividades que estển encaminadas a formar a las docentes y auxiliares.

Jornadas de salud: en cuanto a las jornadas de salud es importante la periodicidad de las mismas, la formación y competitividad del personal responsable de atender a los niños y 
niñas, la disponibilidad e higiene de materiales que se implementen durante estas jornadas y la asistencia de todos los menores a estas jornadas.

Uso de materiales: si bien, el operador del servicio suministra a las docentes y auxiliares de las sedes, una serie de materiales y recursos para el desarrolo de las actividades, es importante que se realice seguimiento permanente a la disponibilidad, entrega oportuna y uso adecuado de los materiales en las aulas de los centros infantiles.

De igual forma, podrấ hacer seguimiento, asesoría y evaluación a actividades relacionadas con el suministro de alimentos, la așistencia de los niños y las niñas al Centro Infantil y cualquier otra actividad o proceso de orden institucional que consideren pertinente.

\section{REFERENTE BIBLIOGRÁFICO}

- Colombia: Instituto Colombiano de Bienestar Familiar. Acuerdo 19 de 1993 (abril 21). (en línea). Disponible en: http://www.icbf.gov.co/cargues/avance/docs/acuerdo_icbf_0019_1993.htm (Recuperado el 09 de marzo de 2013)

- Aguilar, Luís F. (1993a). Problemas Públicos y agenda de Gobierno. México: Miguel Ángel Porrúa.

- Aguilar, Luís F. (1993b). Problemas Públicos y agenda de Gobierno. México: Miguel Angel Porrúa.

- Aguilar, Luís F. (1993c). Problemas Públicos y agenda de Gobierno. México: Miguel Angel Porrúa.

- ASAMBLEA GENERAL DE LAS NACIONES UNIDAS. (1959). Declaración Universal de los Derechos del niño. (en línea). Disponible en: http://www.juridicas.unam.mx/publica/librev/rev/derhum/cont/4/pr/pr20.pdf (Recuperado el 12 de septiembre de 2012)

- ASAMBLEA GENERAL DE LAS NACIONES UNIDAS. (1989). Convención de los Derechos del niño. (en línea). Disponible en: http://www2.ohchr.org/spanish/law/crchtm. (Recuperado el 12 de septiembre de 2012)

- Constitución Política de Colombia. Congreso de la República de Colombia.

Colombia: Ministerio de Educación Nacional. Decreto 088 de 1976 (enero 22). Por el cual se estructura el Sistema Educativo y se organiza el Ministerio de Educación Nacional. (en línea). Disponible en:

http://www.mineducacion.gov.co/ló21/article-102584.html (Recuperado el 23 de agosto cie 2012)

- Colombia: Ministerio de Educación Nacional. Decreto 1002 de 1984 (abril 24). Por el cual se establece el Plan de Estudios Para la Educación Preescolar, Básica (Primaria y Secundaria) 
y Media Vocacional de 日 Educación Formal Colombiana. (en línea). Disponible en: http://www.mineducacion.gov.co/1621/articles-103663_archivo_pdf.pdf (Recuperado el 02 de agosto de 2012)

- Colombia: Conpes. Documento Conpes 109 de 2007. Política Pública de Primera Infancia: Colombia por la primera inf ancia.

- Colombia: Conpes. Documento Conpes 2787 de 1995. Política Pública sobre la infancia: el tiempo de los niños.

- Elmore, R (1982). Backward Mapping: Implementation research and Policy decision. New Jersey: Jstor.

- Henríquez Algarín, H. (2013). Políticas Públicas y desarrollo en educación. Universidad del Magdalena. Colombia.

- Kornblit. Ana L. (2007). Metodologías Cualitativas en Ciencias Sociales: Modelos y procedimientos de análisis. Segunda edición. Buenos aires: Biblos.

- Larrue, C. (2000). Analizar el contexto de la Política. París: L'Harmattan.

- Meny, I. yThoenig, J.C. (1992). Las Políticas Públicas, (trad.), Barcelona: Ariel.

- Ministerio de Desarrollo Social: Chile CRECE contigo. (2012). Sistema de Protección Integral a la Infancia. (en línea). Disponible en: http://www.crececontigo.gob.ci/ (Recuperado el 26 de marzo de 2013).

- Ministerio de Educación y Deportes. (2004). Proyecto Simoncito. Educación inicial de calidad Política de atención integral para los niños y niñas entre cero y seis años. Venezuela: Educere v.11 No.36.

- Colombia: Ministerio de Educación Nacional. (2010). Guía operativa para la prestación del servicio de atención integral a la Primera Inf ancia. Colombia: MEN.

- Muller, P. (2000). Las Políticas Públicas. (en línea). Colombia: Universidad Externado de Colombia. Disponible en:

http://cursodeposgrado.files.wordpress.com/2011/08/muller.pdf

(Recuperado el 04 de diciembre de 2012).

- Colombia: Congreso de la República, Ley 75 de 190 (30 de diciembre). Por la cual se dictan normas sobre filiación y se crea el Instituto Colombiano de Bienestar Familiar. (en línea). Disponible en:

http://www.alcaldiabogota.gov.co/sisjur/normas/Norma 1.jpp?i=4828

(Recuperado et 12 de agosto de 2012).

- Colombia: Gobierno Nacional. Ley 27 de 1974 (20 de diciembre). Por la cual se dictan normas sobre la creación y sostenimiento de Centros de Atención Integral al Pre-escolar. 
para los hijos de empleados y trabajadores de los sectores públicos y privados. Colombia: Gobierno Nacional. (en línea). Disponible en: http://www.alcaldiabogota.gov.co/sisjur/normas/Norma1 .jsp?i=10580 (Recuperado el 23 de agosto de 2012).

- Colombia: Congreso de la República. Ley 100 de 1993. (diciembre 23). Por la cual se crea et sistema de seguridad social integra.

- Colombia: Ministerio de Educación Nacional. Ley 115 de 1994. Por medio de la cual se expide la Ley General de Educación.

- Colombia: Departamento Nacional de Planeación. Plan Nacional de Desarrollo Social, Económico y Regional 1974 - 1978. (en línea) Disponible en: http://www.lib.utexas.edu/ benson/lagovdocs/colombia/federal/planeacion/PND-1974-1978.pdf (Recuperado el 23 de agosto de 2012)

- Plan Nacional de Desarrollo 1982 y 1986: Cambio con Equidad. Colombia: Departamento Nacional de Planeación. (en línea). Disponible en: http://www.lib.utexas.edu/benson/lagovdocs/colombia/federal/planeacion/PND-1982-1986.pdf (Recuperado el 02 de agosto de 2012)

- Organización de las Naciones Unidas para la Educación la Ciencia y la Cultura. (2000). Objetivos de Desarrollo del Milenio. (en línea). Disponible en: http://www.un.org/es/millenniumgoals/. (Recuperado el 12 de septiembre de 2012).

- Organización de las Naciones Unidas para la Educación la Ciencia y la Cultura. (2000). Educación para todos, cumplir nuestros compromisos. (en línea). Disponible en: http:// www.unesco.org/new/es/our-priorities/education-for-all/. (Recuperado el 29 de septiembre de 2012).

- Organización de las Naciones Unidas para la Educación la Ciencia y la Cultura. (2007). Informe de seguimiento a la ET, atención y educación de la Primera Infancia. (en línea). Disponible en: http://unesdoc.unesco.org/images/0015/001505/F505185.pdf. (Recuperado el 2 de julio de 2012).

- Organización de las Naciones Unidas para la Educación, la Ciencia y la Cultura. (año). Conferencia de Moscú: Construir la riqueza de las naciones. (en línea). Disponible en: http://www.unesco.org/new/es/media-services/single-view/news/early_child hood_ care_and_education_the_earlier_the_better/. (Recuperado el 9 de marzo de 2012).

- Roth, A. (2006). Políticas Públicas: formulación, implementación y evaluación. Bogotá: Aurora.

- Salazar, C (20 12a). De la evaluación al análisis de políticas públicas. Madrid: Academia Española. 
- Salazar, C (1993b). Modelos para el análisis de políticas públicas. Bogotá: Pontificia Universidad Javeriana.

- Sandoval, Carlos A. (1996), Investigación Cualitativa. ICFES. Colombia.

- Tamayo, M. (1997). E análisis de las políticas públicas. Madrid: Alianza Universidad textos.

- Unicef. (2008). Fondo de las Naciones Unidas para la Infancia. (en línea). Disponible en: http://www.unicef.org/spanish/childsurvival/index_34322.html. (Recuperado el 23 de Noviembre de 2012).

- Unicef. (2001). Estado mundial de la Infancia 2001 (pdf). (en línea). Disponible en: http://www.unicef.org/span ish/publications/files/pub_sowc0 1_sp.pdf. (Recuperado et 10de Diciembre de 2012).

- Unicef. (2012). Estado Mundial de la Infancia 2012 (pdf). (en línea). Disponible en: http://www.unicef.org/span ish/sowc2012/pdfs/SOWC\%202012\%20Main\%20Report\%20LoRes\%20PDF_SP_03132012.pdf. (Recuperado el 10 de Diciembre de 2012).

- Unicef. (2012). Programa Ciudades Amigas de la Infancia. (en línea). Disponible en: http:// www.ciudadesamigas.org/programa.html. (Recuperado el 6 de septiembre de 2012). 


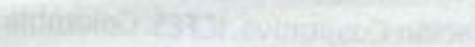

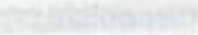
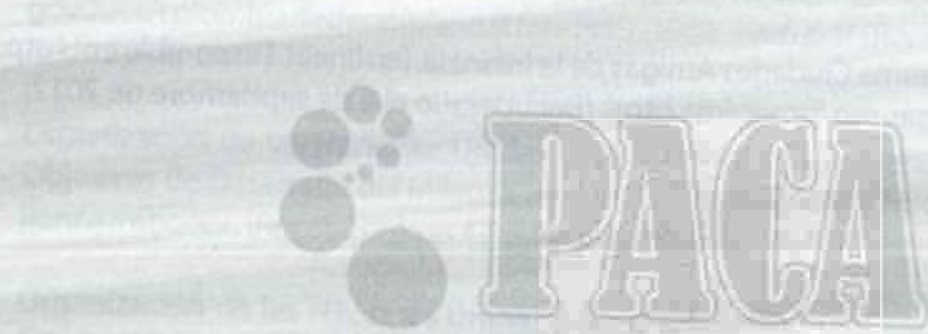

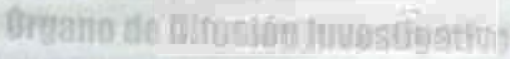

